\title{
When more is less: Negative exposure effects in unsupervised learning
}

\author{
JOHN P. CLAPPER \\ California State University, San Bernardino, California
}

\begin{abstract}
In this article, two broad classes of models of unsupervised learning are compared: correlation tracking models, according to which learning is expected to increase monotonically with exposure to instances, and category invention models, which can accommodate specific violations of monotonicity (negative exposure effects). In two experiments, increasing the number of training instances had a negative rather than a positive effect on unsupervised learning, a clear violation of monotonicity. The results of these experiments are then compared with the predictions of two computational models, one a category invention model and the other a correlation tracking model. The category invention model was able to reproduce the qualitative pattern of results from the human data, whereas the correlation tracking model was not. Overall, these results provide strong evidence for the existence of a discrete category invention process in unsupervised learning.
\end{abstract}

Discovering new categories through unguided observation in the absence of explicit feedback or reinforcement is a fundamental ability that underlies much of human learning (see, e.g., Billman \& Knutson, 1996; Clapper \& Bower, 1991, 1994, 2002; Mervis, 1987; Murphy, 2002; Nelson, 1974). This kind of self-directed pattern detection is often referred to as observational or unsupervised learning, in contrast to supervised learning, in which categories are defined in advance and participants receive corrective feedback from an external teacher (see, e.g., Bruner, Goodnow, \& Austin, 1967; Medin \& Schaffer, 1978; Posner \& Keele, 1968, 1970; Smith \& Medin, 1981). In this article, an attempt is made to provide a test to discriminate between two major theories-or, more accurately, two classes of theories - of human unsupervised learning.

Several models of unsupervised learning (see, e.g., J. R. Anderson, 1990, 1991; Clapper \& Bower, 1991; Gureckis \& Love, 2003; Love, Medin, \& Gureckis, 2004; Schank, 1982) suggest that new categories are created in response to expectation failure, novelty, surprise, or mismatchthat is, people create new categories in response to stimuli that do not fit their existing categories. Such category invention is generally conceived as a discrete, all-or-none mental operation triggered by match failure and accompanied by increased attention and a subjective recognition that the present stimulus is "something different"-a sort of internal "click" or orienting response that marks the recognition of a new type of object. To illustrate, imagine

Correspondence concerning this article should be addressed to J. P. Clapper, Department of Psychology, California State University, 5500 University Parkway, San Bernardino, CA 92407-2397 (e-mail: jclappe@ csusb.edu)

Note-This article was accepted by the previous editorial team, when Colin M. MacLeod was Editor. showing a completely unfamiliar creature, perhaps a new and unusual type of tropical invertebrate, to a person with no prior knowledge of this animal. Commonsense intuition suggests that the person would immediately recognize this creature as new and unfamiliar, assume that the specific individual they saw was an example drawn from a larger class of similar creatures, and attempt to fit this new class into their existing system of knowledge (e.g., if the creature looked somewhat bug-like, they might infer that it is a type of insect or spider). The accompanying sense of novelty and surprise would help draw attention to the new category and provide the cognitive resources needed to integrate it into the person's existing knowledge base (Clapper \& Bower, 1991; Schank, 1982).

There is an obvious alternative to the "sudden insight" position, however. This alternative position assumes that categories emerge gradually, much as described by the classic doctrine of the association of elements (see, e.g., Bain, 1855/1968; Hume, 1739/2000). Within this framework, stimuli are conceived as collections of features and the mind as a mechanism that tracks how often different features occur together over time. This kind of correlation tracking provides much the same predictive information as creating explicit categories - that is, given that some features of an object are known, it becomes possible to predict its remaining features with greater-than-chance accuracy. There are many examples of this type of model in the psychological literature, such as the rule sampling models of Zeaman and House (1963), Davis (1985), and Billman and Heit (1988), as well as various single-layer connectionist autoassociation models (e.g., J. A. Anderson, 1977; J. A. Anderson, Silverstein, Ritz, \& Jones, 1977; Kohonen, Oja, \& Lehtio, 1981; McClelland \& Rumelhart, 1985). Such models are surprisingly powerful and can account for a wide variety of phenomena related to unsupervised learning. Obviously, there is no need to assume that 
people invent explicit categories if their behavior can be explained by such a simple feature association process; hence, correlation tracking provides a useful null hypothesis for evaluating claims about category invention.

This article will be organized as follows: First, it will be argued that one important difference between these two frameworks is the way in which learning is assumed to depend on exposure to specific instances. Specifically, correlation tracking models expect learning to increase as a strictly monotonic function of exposure, whereas category invention models can accommodate specific violations of monotonicity. Second, this difference will be used to motivate several predictions about human learning performance, and two experiments in which those predictions are tested will be reported. Finally, the logical arguments advanced in the first part of the article will be extended by investigating how two contrasting computational models - one a representative category invention model and the other a representative correlation tracking model- perform when given the training sequences used in the experiments. The theoretical implications of these results will be discussed at the end of the article.

\section{Monotonicity and Models of Learning}

In addition to differing in the types of computations involved in unsupervised learning and the nature of the resulting mental representations, category invention and correlation tracking models differ sharply in their views of how learning (or knowledge) is related to practice and experience. In particular, the correlation tracking perspective suggests that learning should be an incremental, monotonic growth process in which each repetition of a given pattern etches that pattern (or at least some of the correlations that comprise it) more deeply into memory (Clapper \& Bower, 2002). Learning of a pattern can only increase, and never systematically decrease, as a function of additional exposure to that pattern (all else being equal). In principle, this framework would appear to imply that people should eventually learn all the correlational structure in their environment, given sufficient time and exposure (assuming that they perceive and attend to the features that participate in those correlations).

This commitment to monotonicity is not shared by category invention models. These models are defined by two basic assumptions about the process of unsupervised learning (Clapper \& Bower, 1991). The first assumption is that the learner attempts to assign each presented stimulus to an existing category; if no existing category provides an adequate match for a given stimulus, then a new category is generated to cover or describe that stimulus. Note that this implies a kind of "win-stay-lose-shift" learning strategy (Trabasso \& Bower, 1968) in which new categories are created only in response to the failure of old ones. It also implies a dichotomous, or all-or-none, conception of learning in which stimuli are either partitioned into separate categories or aggregated together into a single category. The second assumption is that norms or beliefs about a category are computed by averaging or aggregating across instances within that category (e.g., comput- ing summary statistics such as probability distributions for each dimension). These norms then define category boundaries when future instances are encountered and provide expectations regarding likely features of such instances.

Under certain conditions, category invention models can exhibit sharp departures from monotonicity. To illustrate, consider an experiment reported by Clapper and Bower (2002; see also Clapper \& Bower, 1994). In that experiment, participants saw a series of training instances (verbal descriptions of fictitious tree species) from two correlational patterns (potential categories), referred to here as A and B. (Participants were not informed about these categories; the task was designed to assess whether or not they would discover the categories for themselves). The stimuli consisted of 12 dimensions, 9 of which had consistent values within a category and 3 of which were free to vary (i.e., Category $\mathrm{A}=111111111 \mathrm{XXX}$ and Category $\mathrm{B}=222222222 \mathrm{XXX}$, where each position represents a dimension, each number a particular value on that dimension, and $\mathrm{X}$ a dimension with a variable value). When several As were shown prior to any Bs, as in the sequence A A A A A A A A A A A A A A A B B A B B A B B B A ..., participants showed good learning of both categories. But when instances of the two categories were presented in a randomly mixed sequence (e.g., A B B A B A A B B A A B B A B A - B A A B B A B B B A ...), participants showed much poorer learning of both. Note that the only difference between the two sequences is that several Bs in the second sequence (indicated here in bold type) took the places of As in the first sequence. Thus, learning of Category B was actually better in the condition in which fewer instances of that category were shown; such a negative exposure effect is a clear violation of monotonicity.

To the extent to which they assume monotonicity, correlation tracking models are ruled out by this result. However, the same result can easily be accommodated within the category invention framework (Clapper \& Bower, 2002). Consider a hypothetical learner exposed to two different training sequences. Given a sequence similar to the first sequence above (referred to as a "contrast sequence" by Clapper \& Bower, 2002), this learner would be expected to create a new category (Category A) at the beginning of the experiment (see Figure 1A) and then acquire general norms about that category by aggregating over the A instances presented during the next several trials. By the time the first B is encountered, the learner should have strong expectations regarding the consistent features of Category A, narrowing the boundaries for acceptance into that category. The B instance, which mismatches several of these consistent features, should by now fall outside the boundaries of the A category. This should trigger the creation of a new category to cover the B instance; subsequent Bs would then be assimilated to the new B category, whereas As would continue to be assigned to the original A category.

The situation should be quite different in the second (mixed) sequence described above. Here, the learner 
(A) AAAAAAAAAAAA BABAABABABAB ...

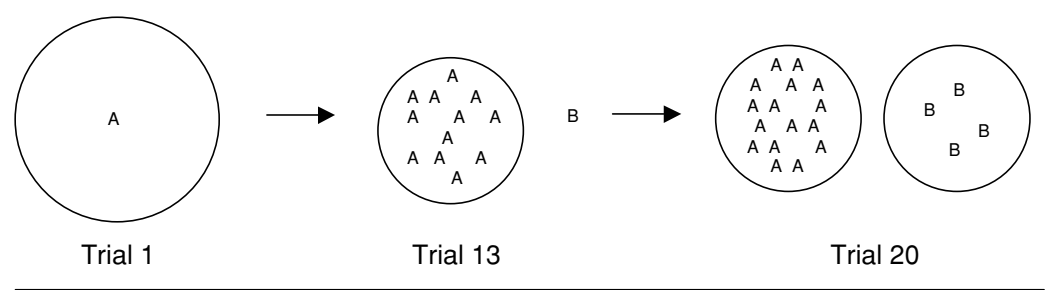

(B) ABBABABAABBA BABAABABABAB ...

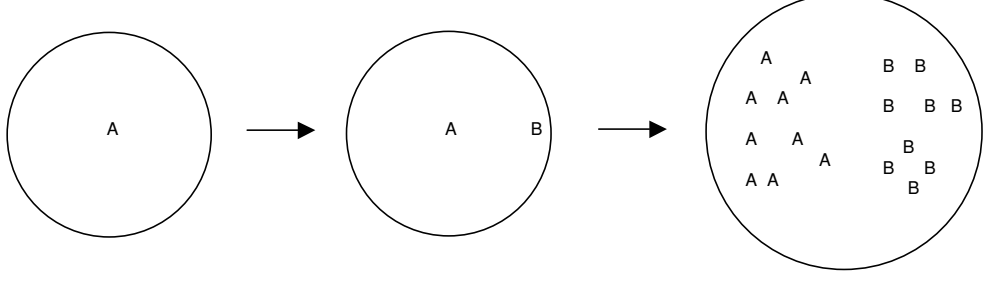

Trial 1

Trial 2

Trial 18

(C) BAABABAB AAAAAAAAAAAAAAAAAAAAAAAA BABAABABABAB ...

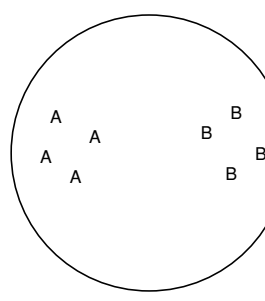

Trial 8

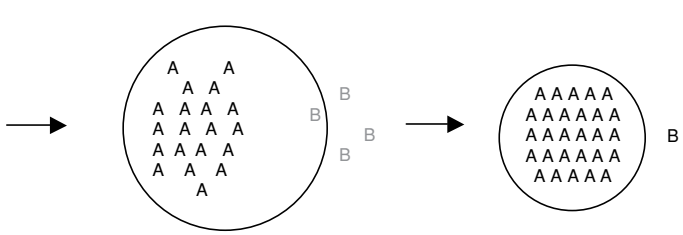

Trial 24

Trial 33

Figure 1. Set diagrams illustrating learning predictions of the category invention model given different training sequences.

would have little time to learn strong A norms before encountering the first B instance. At this point, the boundaries of the A category might still be sufficiently flexible to allow the $\mathrm{B}$ instance to be accepted as a member (see Figure 1B). The failure to trigger a separate $\mathrm{B}$ category means that the A and B instances would become lumped together into a single overaggregated category. Once this occurs, the learner should continue to assimilate further instances of both types to the aggregated category. This is because that category continues to provide an acceptable match to both $\mathrm{A}$ and $\mathrm{B}$ instances, and hence there is never any distinct failure event (see, e.g., Schank, 1982) to force the learner to generate separate categories.

This analysis suggests that once two or more categories are aggregated together, the learner should remain trapped in an "unlearned" state that is largely immune to further experience. Obviously, this is a somewhat counterintuitive conclusion. From a pragmatic or adaptive perspective, it would seem that any sensible learning process should eventually be able to capture the majority of the correlational structure in its environment, and that it should not be subject to the kind of rigidity and order sensitivity implied here. Thus, the aggregation phenomenon poses a kind of recovery problem - once aggregation has occurred, how could a category invention process ever recover and go on to internalize the structure of its stimulus set?

In fact, there are certain situations in which a winstay-lose-shift category invention process should be able to recover from this kind of error. Consider the training sequence shown in Figure 1C. Here, learners are first shown several As and Bs in a mixed sequence. Following this, a long series of A-only trials is shown, after which the previous mixed sequence resumes. Category invention makes two predictions about this sequence. First, learners should aggregate both types of instances into a single category during the early mixed trials, and this should have a negative effect on later learning. Second, it should be possible to overcome this aggregation and learn separate categories, but only given a sufficient number of A-only trials.

Beginning with the second prediction, a long series of A instances should cause the A values to gradually swamp or overwhelm the B values within the aggregated category. Eventually, the boundaries of this category should narrow so much that any B would now appear to be outside those boundaries (middle panel of Figure 1C). In other words, as the $\mathrm{A}$ values grow stronger and the $\mathrm{B}$ values grow weaker, 
a point should finally be reached at which any B instance would appear sufficiently anomalous to trigger a new category (right panel of Figure 1C).

Returning to the first prediction, however, it seems obvious that a longer series of $\mathrm{A}$ instances would be required to reach the triggering threshold (i.e., the point at which a $\mathrm{B}$ instance evokes a new category) in the sequence shown in Figure 1C than in that shown in Figure 1A. This follows from the fact that the A values must overcome their initial aggregation with the $\mathrm{B}$ values before the triggering threshold can be reached. Thus, a series of A instances long enough to produce good learning in the sequence of Figure 1A may not be long enough to do so in the sequence of Figure $1 \mathrm{C}$, due to the interfering effects of prior aggregation.

The aim of the two experiments reported below was to test these predictions. The task used in these experiments was the sequential unsupervised learning task introduced by Clapper and Bower (2002). This task is particularly useful for the present type of investigation because (1) it measures incidental unsupervised learning, in the sense that participants are never asked to sort instances or otherwise search for explicit categories (in fact, they are never alerted to the possible existence of separate categories); and (2) it measures learning continuously, on a trial-bytrial basis, unlike many tasks that measure learning only at the conclusion of the experimental procedure.

In this task, participants are presented with a training instance (a verbal feature list) on a computer monitor, are asked to study it for a specified period of time, and then receive a series of forced-choice recognition tests for the features of that instance. This study-test procedure is repeated over a series of trials. Each training instance is displayed in such a manner that the learner can examine only one feature at a time, and the total amount of time spent studying each feature is recorded by the computer. The stimuli typically come from two or more patterns (potential categories) characterized by different clusters of correlated properties (in which some properties are consistently present within a pattern whereas others are free to vary). As participants learn the patterns, they spend more time studying the (uncorrelated) variable features of the training instances and less time studying their (correlated) consistent features; this preference results from the greater uncertainty regarding the current value of an uncorrelated feature once the correlated features are learned (i.e., uncorrelated features have greater "information value" than correlated features for recall of each instance; see, e.g., Metcalfe, 2002). In addition to this increasingly biased allocation of study time, memory for the correlated features of the instances improves because these features become increasingly predictable as people learn the patterns. Memory for uncorrelated attributes may also improve due to the additional study time they receive. Both the study time and the memory measures can be plotted over trials to reveal learning curves for the correlational patterns or categories in the stimuli. In addition, participants' knowledge of the correlational structure of the stimulus set was assessed directly at the end of the experiment by having them rate how often different pairs of features had occurred together during the prior study-test portion.

\section{EXPERIMENT 1}

The aim of this experiment was to test the first prediction mentioned above - namely, that aggregating two categories early in training would interfere with learning to distinguish them later. There were two conditions. In the contrast condition, a sequence similar to that in Figure 1A was presented. In this condition, the first 12 training instances were all members of Category A, after which an equal number of As and Bs were presented in mixed alternation over the next 24 trials. In the aggregation condition, a sequence similar to that in Figure $1 \mathrm{C}$ was presented (except that 12 rather than $24 \mathrm{~A}$ trials followed the initial mixed sequence). This was identical to the sequence used in the contrast condition except that 8 mixed trials, consisting of an equal number of both As and Bs, were added to the beginning of the sequence. Thus, a total of 44 trials was shown in the aggregation condition whereas only 36 trials were used in the contrast condition.

It was predicted that most participants in the contrast condition would create separate categories and readily learn the consistent values within each one. By contrast, participants in the aggregation condition were expected to lump the categories together during the first eight trials. It was expected that the following $12 \mathrm{~A}$ instances would be insufficient for most participants to overcome this initial aggregation. As a result, when B instances were reintroduced later, they would be assigned to the category created earlier rather than trigger a separate new category. The outcome of this continued aggregation would be a failure to learn the consistent features within either category.

It is important that the two conditions were identical except for the fact that several instances of both categories were added to the beginning of the contrast sequence to create the aggregation sequence. Thus, poorer learning in the aggregation condition (in which more instances were shown) would be a clear violation of monotonicity (i.e., a negative exposure effect) for both categories.

\section{Method}

\section{Participants}

Sixteen undergraduate students of California State University at San Bernardino participated in return for extra credit in any of several psychology classes.

\section{Procedure}

The procedure consisted of two phases. In the first, a series of training instances was presented using the unsupervised learning procedure of Clapper and Bower (2002). In the second, participants rated how often different pairs of features had co-occurred in Phase 1.

Unsupervised learning task. At the start of each trial, a verbal list (describing a fictitious species of tree) was presented in the middle of a computer screen. The tree's name was printed at the top of the list, and below it appeared a list of 12 short descriptive phases, each referring to a specific value of a particular attribute of that tree. A different attribute was shown on each row (line) of the list display. 
All the features in the list display were masked by rows of Xs at the beginning of each trial. The participants examined the features by pressing the up-arrow or down-arrow key, which unmasked the feature above or below their current position, respectively (the computer chose a different random starting location on each trial). The exposed descriptor was immediately masked again by Xs when the participants unmasked a new line (descriptor). A total of $24 \mathrm{sec}$ was allocated for the participants to study all the features in the 12-item list. The computer program recorded the total amount of time spent looking at each feature.

Immediately after the list disappeared, the participants received a series of forced-choice recognition tests for the features of this instance. Four test questions were presented, one at a time, in a multiple-choice format. Features were selected randomly for testing with the constraint that three tests should pertain to correlated features and one to uncorrelated features on each trial. All tests were self-paced. The tree's name was shown at the top of the display, and four possible answers (different values of the same attribute) were listed at the bottom. The participants typed in the number (1-4) corresponding to the value that they believed was present in the instance they had just studied. The computer then displayed feedback regarding the accuracy of the response, and if an error occurred the correct response was indicated. Following all the recognition tests for a given instance, the participants received summary feedback on their performance for that instance and on how it compared with their cumulative average over previous trials. A congratulatory message was presented to them if they had surpassed their previous average, and they were encouraged to try to do so on the next trial if they had not.

Correlation rating task. During each trial of this phase, the participants rated how frequently different pairs of features (values of different attribute dimensions) had occurred together in the preceding phase. They used a 5-point scale on which 1 indicated that the features never occurred together; 2 , that they sometimes occurred together; 3 , that they commonly occurred together; 4 , that they usually occurred together; and 5, that they always occurred together. A total of 16 such pairs was shown during the posttesting phase.

\section{Materials}

The instances (tree descriptions) consisted of 12 substitutive attribute dimensions, each with four possible values. Examples of these attributes included the color of the tree's bark (dark gray, deep brown, mossy green, or light tan), its form or overall shape (low and shrub-like, tall and column-like, massive and wide branching, or twisting and vine-like), and the season in which it flowers (spring, summer, winter, or autumn). The trees were given Latin names arbitrarily selected from various plant identification guides.

Nine attributes of the training stimuli had perfectly correlated values whereas the other three attributes were uncorrelated. The patterns of Category A may be denoted as $111111111 \mathrm{XXX}$ and that of Category B as 222222222XXX, where the first nine attributes had correlated values (denoted by $1 \mathrm{~s}$ or $2 \mathrm{~s}$ ) and the last three attributes had uncorrelated values (denoted by Xs). The uncorrelated attributes varied independently through all four values across different instances of a given category. Each value occurred an equal number of times within each category.

\section{Design}

The participants were randomly assigned to two conditions, referred to as the contrast and aggregation conditions ( $n=8$ for each condition). These conditions are defined in terms of the sequence in which training instances were presented. In the contrast sequence, the first 12 trials consisted of all A instances whereas the last 24 trials consisted of an equal number of $\mathrm{A}$ and $\mathrm{B}$ instances in a pseudorandom sequence- that is, $\mathrm{A} A \mathrm{~A} A \mathrm{~A} A \mathrm{~A} A \mathrm{~A} A \mathrm{~A} A \cdot \mathrm{B} A \mathrm{~B} A \mathrm{~A}$ B B B A A B A B A A A B B A A B A B B. (Note that no more than three examples from either category were shown in a row during the mixed trials). The aggregation sequence was identical to the contrast sequence except that an additional 8 mixed trials were added prior to the series of A trials-that is, A B A B B A A B - A A A A A A A A A A A A - B A B A A B B B A A B A B A A A B B A A B A B B. These mixed trials consisted of an equal number of $A s$ and $B s$ shown in a pseudorandom order.

The same ordering of trials was used for all participants within each condition, but the actual stimuli were different because stimulus features were randomly assigned to roles within the experimental design for each participant. The order in which features were presented in the instance lists was also randomized separately (but remained constant throughout the session) for each participant.

Four different types of probes (feature pairs) were shown during the correlation rating phase: (1) pairs of correlated attribute values from the same category, (2) pairs of correlated attribute values from different categories, (3) pairs consisting of one correlated value and one uncorrelated value, and (4) pairs consisting of two uncorrelated values. Four examples of each probe type were used.

\section{Results and Discussion}

\section{Correlation Ratings}

Although several types of feature pairs were used in Phase 2, the only data relevant for assessing pattern learning were the average ratings for pairs of correlated values from the same category minus those for pairs of correlated values from different categories. Since the rating scale ranged from 1 to 5 , the discrimination score (absolute value of the difference) that resulted from subtracting one set of averaged ratings from another could range from 0 (no discrimination) to 4 (maximum discrimination).

The mean discrimination score from the contrast condition was 2.08, and that from the aggregation condition was 0.79 . This score from the contrast condition was significantly greater than zero $[t(7)=4.83, S E=0.43, p=$ $.002]$, and that from the aggregation condition was marginally greater than zero $[t(7)=2.01, S E=0.39, p=$ .084]. Discrimination scores were significantly higher in the contrast condition than in the aggregation condition $[t(14)=2.22, S E=0.58, p=.044]$.

\section{Memory}

Correlated features were remembered better than uncorrelated features in both the contrast condition $[t(7)=$ 4.32, $S E=0.04, p=.003$ ] and the aggregation condition $[t(7)=2.65, S E=0.04, p=.033]$. The recognition data for correlated features are shown in Figure 2.

Recognition performance in the two conditions did not differ during the early A trials. (This was true for both correlated and uncorrelated features.) Following these A trials, performance in both conditions dipped when the first $\mathrm{B}$ instance was introduced, then recovered quickly in the contrast condition but continued to fall in the aggregation condition. When the two conditions were compared over the second half of these mixed trials, the difference was significant for correlated features $[t(14)=3.04, S E=$ $0.07, p=.009]$ and marginally significant for uncorrelated features $[t(14)=2.04, S E=0.08, p=.061]$.

\section{Study Times}

The main index of learning derived from the study time measure was a preference score obtained by subtracting average study time for correlated features from that for 


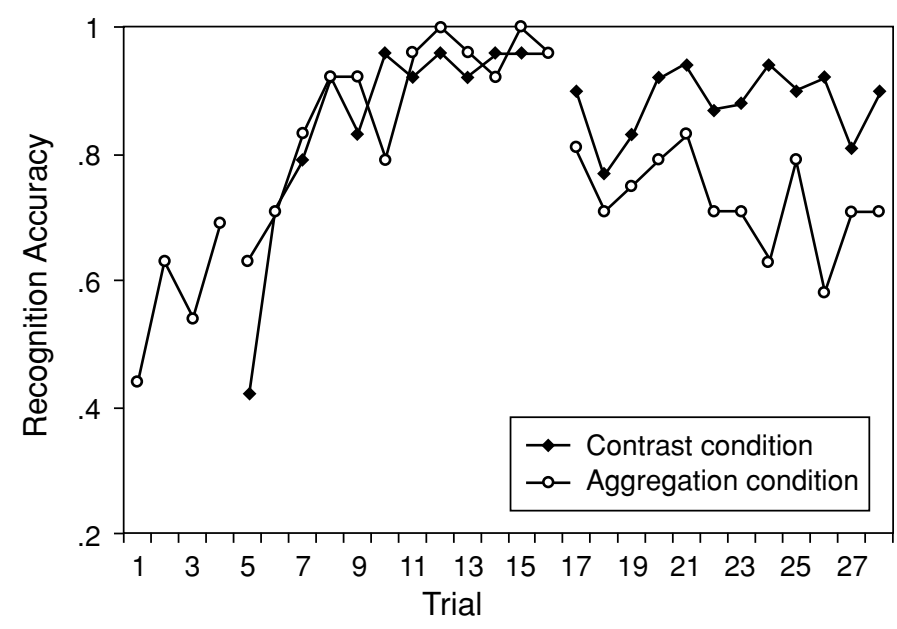

Figure 2. Recognition accuracy for correlated features in Experiment 1 . Note that the curve for the contrast condition has been moved to the right to facilitate comparison with the corresponding trials of the aggregation condition, and that the two categories have been pooled within each condition.

uncorrelated features. Since the participants who had learned the categories were expected to spend more time studying uncorrelated than correlated features, a positive preference score is interpreted as evidence of learning. These data are shown in Figure 3.

Averaged over trials, preference scores were positive in both the contrast condition $[t(7)=6.08, S E=0.11, p=$ $.001]$ and the aggregation condition $[t(7)=3.00, S E=$ $0.09, p=.02]$. Preference scores rose during the A series in both conditions, and there was no significant difference between them during this interval $[t(14)=1.47, S E=$ $0.15, p=.163]$. As Figure 3 shows, preference scores dipped in both conditions when the first $\mathrm{B}$ was introduced following the A series. They increased thereafter in the contrast condition but not in the aggregation condition. The difference between conditions was significant during the second half of this mixed sequence $[t(14)=2.22$, $S E=0.29, p=.043]$.

\section{Conclusions}

The results of this experiment were clear: All three measures indicated better learning in the contrast condition than in the aggregation condition. At first blush, this seems like a very surprising result: Simply adding a few instances of each category to the beginning of a training sequence actually reduced later learning of both catego-

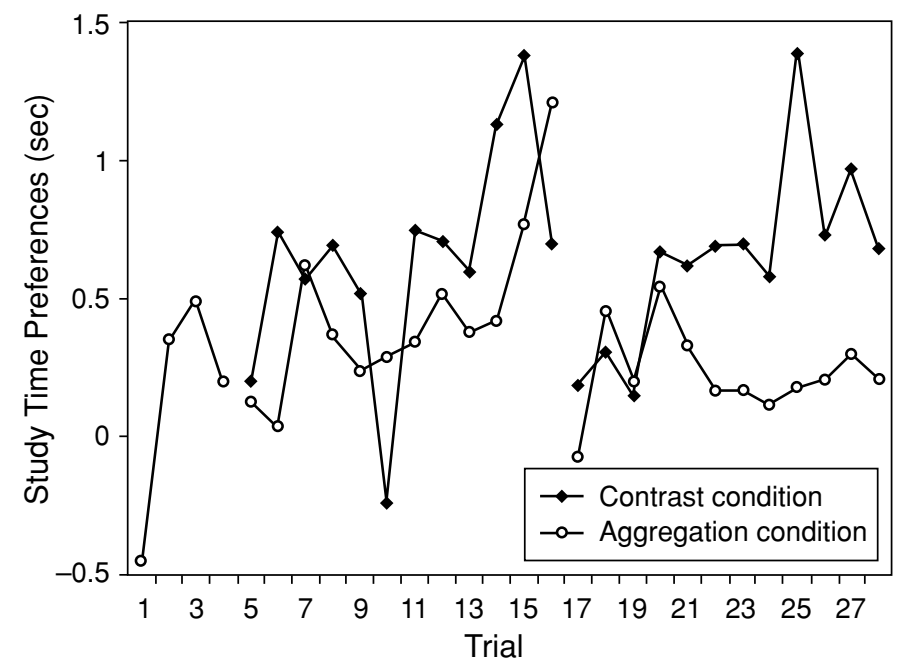

Figure 3. Study time data from Experiment 1. Note that the curves have been adjusted to facilitate comparison between conditions, as in Figure 2. 
ries. A monotonic correlation tracking process cannot accommodate this kind of negative exposure effect. By contrast, these results provide strong evidence for a dichotomous state model of learning - that is, for the kind of win-stay-lose-shift category invention process described in the introduction. Within this framework, exposure is effective only if people have first segregated the instances into their correct categories; if not, exposure to additional instances should be ineffective for teaching them about the correlational structure of these instances.

\section{EXPERIMENT 2}

In this experiment, participants were given a training sequence similar to that of the aggregation condition of Experiment 1, except that the length of the A series was increased from 12 to 24 instances. In theory, a series of all $\mathrm{A}$ instances should gradually strengthen the A values and weaken the B values within the aggregated category. If the series is long enough, the A values should come to dominate the aggregated category to such a degree that any later B instance would seem anomalous and evoke a separate category of its own. Thus, learners in this condition (referred to as the deaggregation condition) should be able to learn separate categories following the A series. In the control (mixed) condition, A and B instances were simply presented in random alternation throughout the experiment. Obviously, little learning should occur in the mixed condition.

It is important that the two conditions differed in only one way: All the B instances within a specific interval of trials in the mixed condition were replaced with $\mathrm{A}$ instances in the deaggregation condition. Thus, the deaggregation sequence was identical to the mixed sequence except that it contained more As and fewer Bs. Hence, superior learning of Category B in the deaggregation condition would constitute another violation of monotonicity. This negative exposure effect would be a further strike against correlation tracking models. (Note that better learning of Category $\mathrm{A}$ in the deaggregation condition would not violate monotonicity, because there were more instances of $\mathrm{A}$ in this condition than in the mixed condition.)

\footnotetext{
Method

Participants. Forty undergraduate students of California State University at San Bernardino participated in return for extra credit in any of several psychology classes.

Procedure, Materials, and Design. The procedure and materials used in this experiment were the same as those in Experiment 1. Each participant was randomly assigned to either the deaggregation condition $(n=22)$ or the mixed condition $(n=18)$. The first 8 and last 24 trials (out of 56) were identical in both conditions. During these trials, an equal number of $\mathrm{A}$ and $\mathrm{B}$ instances was shown in a pseudorandom sequence (i.e., no more than three instances of the same category were allowed to occur in a row). The two conditions differed only in Trials 9-32. In the mixed condition, these trials consisted of an equal number of $\mathrm{A}$ and $\mathrm{B}$ instances, presented in the same type of mixed sequence as in the preceding and following trials-that is, B A A B A B A B - A A B B B A A B A B A B A A B B A B A A B B A B $\cdot$ B A A A B B B A A B A B A A B B A B
}

A A B A B B. In the deaggregation condition, all the B instances were eliminated from these trials and replaced by As, yielding the sequence B A A B A B A B - A A A A A A A A A A A A A A A A A A A A A A A A B A A A B B B A A B A B A A B B A B A A B A $B$ B. (These instances had the same uncorrelated values in the two conditions; only their correlated values were changed.) As in Experiment 1 , the same order of trials was used for all participants, but the actual stimuli they saw were different because stimulus features were randomly assigned to roles within the experimental design for each participant. The same types of probes were used during the correlation rating phase as in Experiment 1.

\section{Results and Discussion}

Correlation ratings. The mean discrimination scores were 2.27 in the deaggregation condition and 0.39 in the mixed condition. Both were significantly greater than zero [deaggregation condition, $t(21)=7.96, S E=0.28$, $p=.000 ;$ mixed condition, $t(17)=2.23, S E=0.17, p=$ .039]. Discrimination scores were significantly greater in the deaggregation condition than in the mixed condition $[t(38)=5.33, S E=0.35, p=.000]$.

As noted, only the results for Category B are informative for evaluating monotonicity in this experiment. In fact, both categories showed better learning in the deaggregation condition [Category A, $t(38)=4.65, S E=$ $0.41, p=.000$; Category B, $t(38)=5.82, S E=0.33, p=$ $.000]$. Thus, the expected violation of monotonicity was confirmed for these data.

Following these analyses, the discrimination scores (pooled over categories) were used to rank order participants from best to worst within each condition. Subsequent analyses were carried out on the top three quarters of learners within each group; the bottom quarter of each group was excluded from the analyses discussed below. This filtering had no effect on the overall pattern of results, but eliminating the poorer (and presumably less motivated) learners reduced noise in the data and increased the reliability of the statistical tests.

Memory. Correlated features were remembered better than uncorrelated features in both the deaggregated condition $[t(16)=4.60, S E=0.03, p=.000]$ and the mixed condition $[t(12)=4.76, S E=0.04, p=.000]$. The recognition data for correlated features are shown in Figure 4.

As was expected, there were no differences between conditions over the first eight trials, since these were identical in both groups. Following these early trials, memory for correlated features increased during Trials 9-32 in the deaggregation condition but not in the mixed condition. Memory averaged over this interval was significantly greater in the deaggregation condition for correlated features $[t(38)=7.43, S E=0.03, p=.000]$ and for uncorrelated features $[t(38)=2.13, S E=0.05, p=.04]$.

Turning to the last 24 mixed trials, memory for correlated features was significantly greater in the deaggregation condition than in the mixed condition $[M=0.93$ vs. $M=0.73 ; t(28)=3.92, S E=0.05, p=.001]$. The same was true for uncorrelated features $[M=0.78$ vs. $M=0.57 ; t(28)=2.56, S E=0.08, p=.016]$. When the results for the two categories were analyzed separately, it was found that the correlated features of Category B were 


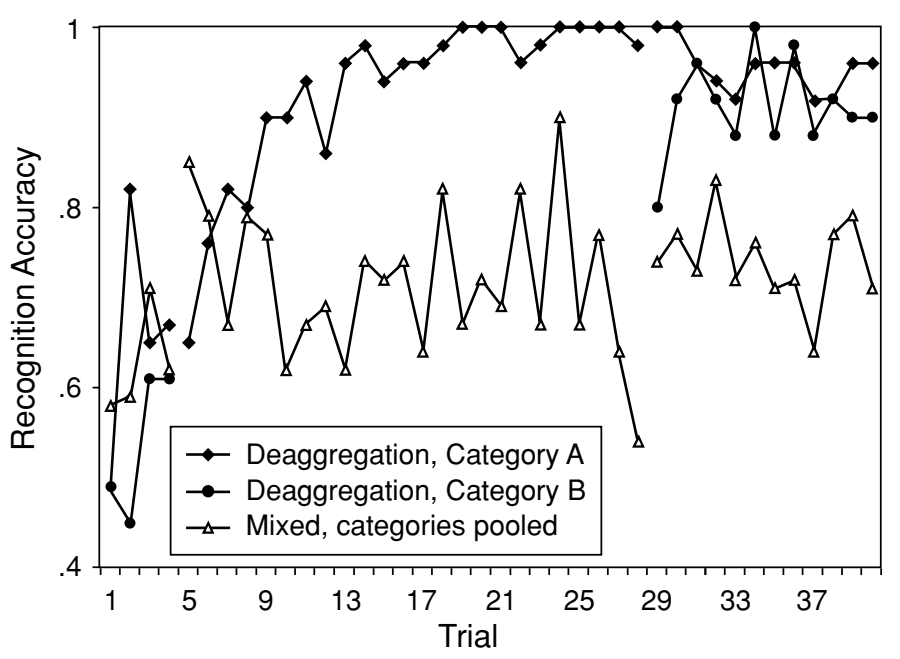

Figure 4. Recognition accuracy for correlated features (top $75 \%$ of learners) in Experiment 2.

remembered better in the deaggregation condition than in the mixed condition $[t(28)=2.76, S E=0.06, p=.01]$. The difference was marginally significant for uncorrelated features $[t(28)=1.86, S E=0.10, p=.074]$. Category A showed the same differences [correlated features, $t(28)=$ 4.91, $S E=0.05, p=.000$; uncorrelated features, $t(28)=$ 3.04, $S E=0.08, p=.005]$.

Study times. The study time data are shown in Figure 5. As was expected, there were no differences over the first eight trials. Preference scores began to climb during the target interval for the deaggregation group but not for the mixed group. Preference scores in the deaggregation condition averaged $0.82 \mathrm{sec}$ during the second half of the target interval, in comparison with $0.04 \mathrm{sec}$ in the mixed condition $[t(28)=2.50, S E=0.31, p=.018]$.
The difference between these conditions was also significant during the final mixed trials $[t(28)=2.20, S E=$ $0.23, p=.036]$. When the categories were analyzed separately over the second half of this series (Trials 44-45), the difference was significant for both Category $\mathrm{A}[t(28)=$ $2.72, S E=0.32, p=.011]$ and Category B $[t(28)=2.41$, $S E=0.28, p=.023]$.

Conclusions. The predicted pattern of results was confirmed by this data. First, the fact that Category B was learned better in the deaggregation than in the mixed condition is a violation of monotonicity, which provides evidence in favor of category invention and against correlation tracking. Second, the results in the deaggregation condition showed that the participants were able to overcome their initial aggregation and learn separate categories,

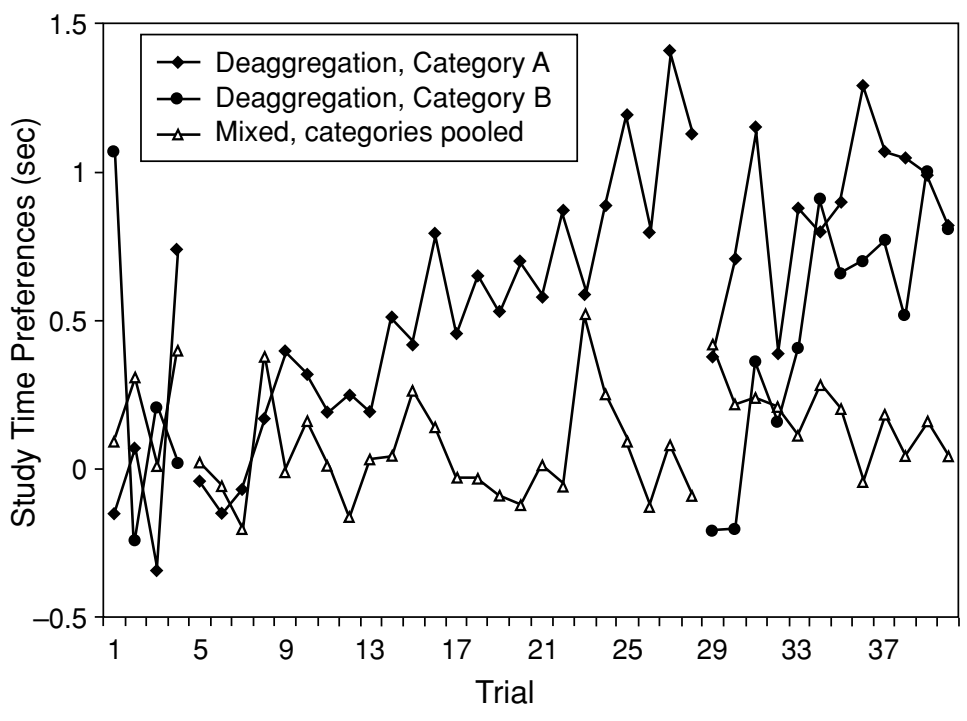

Figure 5. Study time data (top 75\% of learners) from Experiment 2. 
given a sufficiently long sequence of A trials following the earlier mixed sequence. This provides a partial solution to the recovery problem posed in the introduction.

\section{GENERAL DISCUSSION}

In sum, the results of these two experiments were exactly as predicted by the category invention framework: There was persistent interference due to early aggregation of Categories A and B, which could later be reversed with enough isolated exposure to Category A. The violations of monotonicity (negative exposure effects) encountered in both of these experiments are incompatible with correlation tracking but appear to be quite compatible with category invention. Overall, the results clearly support the hypothesis that some sort of all-or-none category invention process plays an important role in unsupervised learning.

The next section will focus on two specific computational models of unsupervised learning, one a well-known category invention model and the other a prominent correlation tracking model. Each model was implemented as a computer simulation and tested under the same conditions used in the present experiments. Comparison of the output of these simulations with the human data provides a useful way to evaluate the models and, possibly, to learn more about the larger classes of models to which they belong.

\section{Computational Models: A Comparison}

The first step in carrying out this type of comparison is to select a representative example of each type of model. J. R. Anderson's $(1990,1991)$ rational model of categorization will be used to represent the category invention approach. This model provides a description of an "optimal" category learning process within the framework of Bayesian probability theory. This claim to normative optimality, along with its relative simplicity and transparency, makes the rational model a natural choice to represent the category invention approach. A version of this model, modified to account for human memory limitations, was used by Clapper and Bower (2002) to model data similar to those of the present experiment.

Selecting a representative correlation tracking model presents a choice between rule sampling models (see, e.g., Billman \& Heit, 1988; Davis, 1985; Zeaman \& House, 1963) and connectionist autoassociation models (see, e.g., J. A. Anderson, 1977; J. A. Anderson et al., 1977; Kohonen et al., 1981; McClelland \& Rumelhart, 1985). Autoassociation models (single-layer networks in which all elements are connected to each other and which learn by strengthening connections between consistently cooccurring elements) are particularly attractive for the present purposes due to their relatively realistic assumptions about learning and memory. For example, the superpositional nature of memory in these models allows them to reproduce some of the interference effects shown in the present data. The autoassociation model described in McClelland and Rumelhart (1985) will be used for the present comparison. This influential model has been shown to account for a number of important findings in the categorization and memory literatures (see, e.g., McClelland \& Rumelhart, 1985).

The rational model. The primary goal of the rational model is to predict the current value of any dimension of a stimulus, given its other values. To do this, it first attempts to assign each stimulus it encounters to some existing category. The probability that a stimulus belongs to a particular category is computed as a Bayesian posterior probability:

$$
P_{k}=P(k \mid F)=\frac{P(k) P(F \mid k)}{\sum_{k}[P(k) P(F \mid k)]},
$$

where $P(k \mid F)$ is the probability of category $k$ given the feature set $F$ of the current instance, $P(k)$ is the prior probability of category $k$, and $P(F \mid k)$ is the probability of instance $F$ given category $k$. If no existing category matches the current stimulus (i.e., if the null category, which assumes an equal probability of all values within each dimension, is a better match to the current instance than any existing category), then a new category is created to accommodate that stimulus.

The prior probability of a category is a function of how often it has been encountered to date. For known categories,

$$
P(k)=\frac{c n_{k}}{(1-c)+c n},
$$

where $n_{k}$ is the number of prior instances assigned to $k, n$ is the total number of stimuli seen, and $c$ is a parameter referred to as the coupling probability. This is the probability that a randomly sampled pair of instances will come from the same category, which in turn depends on the number of separate categories within the stimulus set (i.e., $c$ provides an estimate of the likelihood that separate categories exist in the current set). The probability of a new category is

$$
P(\mathrm{New})=\frac{(1-c)}{(1-c)+c n},
$$

which is equal to one minus the sum of the $P(k) \mathrm{s}$ for known categories.

The second element in Equation 1 is the conditional probability of the current instance $F$ given category $k$. This is computed as

$$
P(F \mid k)=\prod_{i} P_{i}(j \mid k),
$$

where $P_{i}(j \mid k)$ is the probability of value $j$ of attribute $i$ within category $k$. This depends on how often value $j$ has been encountered within category $k$, combined with prior expectations concerning the probability of $j$ within $k$, as follows:

$$
P_{i}(j \mid k)=\frac{n_{j}+\alpha_{j}}{n_{k}+\alpha_{k}} .
$$

Here, $n_{k}$ is the number of objects previously seen from category $k, n_{j}$ is the number of these objects that had value $j$ on dimension $i, \alpha_{j}$ is the prior expectedness of $j$, and $\alpha_{k}$ is 
the sum of all $\alpha_{j}$ s for attribute $i$. Thus, the subjective probability of a given feature is a function of both empirical observation and prior belief.

In prior applications, it has usually been assumed that memory for training instances is perfect, but the present application requires an explicit consideration of limited memory. One way to include memory limitations within the model is to add an explicit memory parameter (Clapper \& Bower, 2002) to Equation 5 as follows:

$$
P_{i}(j \mid k)=\frac{\delta n_{j}+\alpha_{j}}{\delta n_{k}+\alpha_{k}} .
$$

Here, $\delta$ is a memory parameter that ranges from 0 (no memory) to 1 (perfect memory) and has the effect of reducing the impact of observed instances relative to the model's feature priors. An essentially equivalent (but less transparent) way to capture limited memory would be simply to increase the values $\alpha_{j}$ and $\alpha_{k}$ in Equation 5 while leaving the ratio of $\alpha_{j}$ to $\alpha_{k}$ constant. This would have the effect of weighing the feature priors more heavily relative to observed instances in computing $P(j \mid k)$, thereby simulating the effects of reduced memory.

For the present simulations, the parameter values were set to $c=.90$ and $\delta=.40$. These values were used in previous simulations reported in Clapper and Bower (2002) and are within the range of values shown to be appropriate for simulating human performance in the present task.

In relating this model to the present results, it is assumed that the probability of correctly recalling the current value of a given dimension is a function of that value's probability within the best matching category (i.e., highprobability features will be recalled more accurately than low-probability features). ${ }^{1}$ If the model fails to segregate the training stimuli into separate categories, they will all be assigned to the same category. The probability distribution for each dimension within that category will then reflect the base rate probabilities of each value within the stimulus set as a whole. If the model does segregate the instances into appropriate categories, then the probability distributions within each category will be computed across the subset of instances assigned to that category (i.e., categories are disjunctive partitions of the stimulus set, and norms for each category are computed exclusively within that category).

The autoassociation model. Like the rational model, McClelland and Rumelhart's (1985) autoassociation model processes a series of training instances and updates its current knowledge state in response to each instance. A particular input (instance) activates a pattern of neural units; this activation then reverberates throughout the network for several processing cycles. Once the network has settled down into a stable state, this state is compared with the "target" state. The network output should be the same as the input, unless there are missing features in the input, in which case the model should automatically fill them in (as for the rational model, feature prediction is the primary goal of this model). Connection weights are incremented or decremented as a function of whether a particular unit produces an output that is stronger or one that is weaker than the target output. The result is that connection weights between units (features) that co-occur frequently tend to become stronger over time, whereas those between units that do not co-occur tend to become weak or negative.

The key assumptions of the model concern the activation function for the individual units and the rules for updating connections between the units. Beginning with the activation function, the model assumes that each unit receives input from the external stimulus as well as from other units in the network. The net input, $n_{i}$, to any unit $i$ at a given point in time (processing cycle) is

$$
n_{i}=i_{i}+e_{i}
$$

where $e_{i}$ refers to the external input ( -1 indicates that an element is absent, +1 that it is present, and 0 indicates a missing element) and $i_{i}$ refers to the summed input from all other units $j$ in the network - that is,

$$
i_{i}=\sum_{j} i_{i j} \text {. }
$$

The input to $i$ from a specific unit $j$ is simply

$$
i_{i j}=a_{j} w_{i j},
$$

where $a_{j}$ refers to the activation level of unit $j$ and $w_{i j}$ is the weight (strength) of the connection from $j$ to $i$. Information about feature correlations within a stimulus set are stored in terms of these connection weights.

The activation of a unit during any particular processing cycle is a function of its activation during the previous cycle and the net input during the current cycle, plus parameters $E$ and $D$, which refer to excitation and decay rates, respectively (and which are the same for all units in the network). If the net input to unit $i$ is positive, the change in activation level, $d$, is expressed by the following formula:

$$
d_{i}=E n_{i}\left(1-a_{i}\right)-D a_{i}
$$

If the input to $i$ is negative or zero, the change in activation is

$$
d_{i}=E n_{i}\left[a_{i}-(-1)\right]-D a_{i} .
$$

On a particular trial, a training pattern is presented to the network and the activation level of each unit will be adjusted over multiple processing cycles (50 in the current simulation) until the activation of the units stabilizes. Connection weights are adjusted at the end of each trial according to the difference $\left(\Delta_{i}\right)$ between the internal input $i_{i}$ to each unit $i$ at the end of a trial and the external input $e_{i}$ :

$$
\Delta_{i}=e_{i}-i_{i} .
$$

In other words, each unit computes the difference between the pattern of activation produced by the network and the pattern of activation corresponding to the external stimulus (which plays the role of target pattern for the purposes of training the network).

The input weights to unit $i$ are then adjusted according to the formula

$$
\Delta w_{i j}=S \Delta_{i} a_{j}
$$


where $\Delta w_{i j}$ is the change in the strength of the connection from $j$ to $i$ and $S$ is a global parameter that controls the size of the weight adjustment on each trial. This learning rule is often referred to as the delta rule (see, e.g., Sutton $\&$ Barto, 1981) and is a common weight adjustment procedure in neural network modeling. The values assigned to the variable parameters in these simulations were $E=$ $0.01, D=0.01$, and $S=0.025$.

As has been noted, the input to this model is a vector of activation values that represent the current stimulus. Each dimension is represented by a collection of units, with different units corresponding to different values and one unit shared by all values of the dimension. Thus, a four-value dimension is represented by five units. To represent a particular value of this dimension, two of the units would have activation values of +1 and three would have values of -1 ; for example, Value 1 would be represented as $(++---)$, Value 2 as $(+-+--)$, and so on. Note that this representation is somewhat different from that of McClelland and Rumelhart (1985), who assumed binary dimensions represented by values of -1 or +1 on a single unit.

Within the network, the association of each unit with itself is clamped at zero but the weights of all other connections can be modified by experience. Given an input stimulus, the activation of the corresponding values in the model's output provides a good proxy for accuracy of memory for those values (i.e., features with higher activation levels are assumed to be recalled better).
Comparing the models. The outputs of the two models in comparison with relevant human data from the two experiments of the present study and that of Clapper and Bower (2002) are shown in Figure 6. (Only data for Category B are shown, since that is the category for which each experiment provides a critical test of monotonicity.) The left column in this figure shows results from a simple contrast-versus-mixed-sequence comparison (described in detail in the introduction; see also Clapper \& Bower, 1994, 2002). The top panel in this column shows the human data, whereas the middle panel shows results for the rational model. The two patterns are similar and show strong learning of Category B in the contrast condition and much weaker learning in the mixed condition. In the contrast condition, the rational model encounters an initial series of $\mathrm{A}$ instances and assigns them all to the same category. When it then encounters the first B instance, it creates a new category for this instance. Thereafter, A and $\mathrm{B}$ are assigned to separate categories, and so the model is able to capture the predictive structure (consistent features) within each category. Within the mixed condition, the model encounters the first B after seeing only one A; at this point, the $\mathrm{A}$ values are not strongly expected within the category because only one instance has been learned. Therefore, the B stimulus is assigned to the same category as the previous A stimulus. Both A and B stimuli continue to be assigned to this aggregated category on subsequent trials; hence, separate categories are never created and the
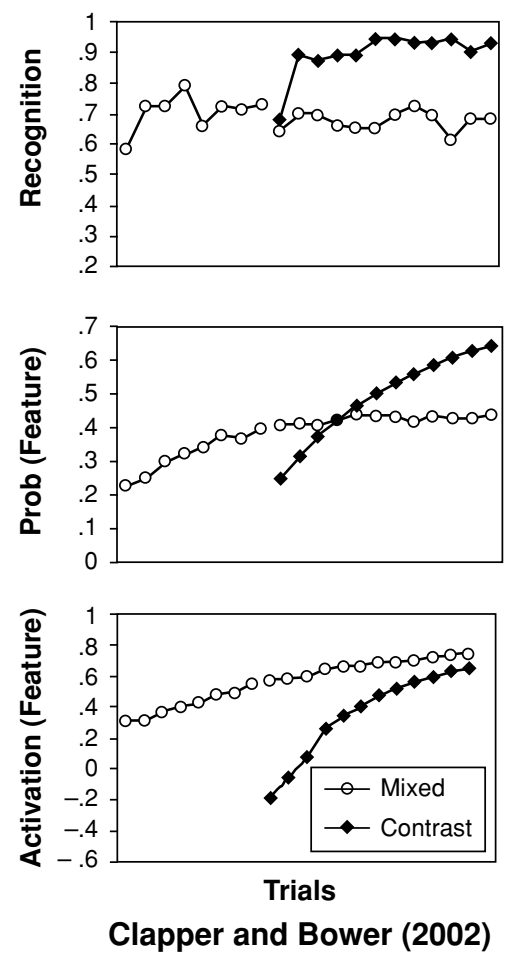

Figure 6. Predicted results for the rational (middle row) and the autoassociation (bottom row) models in comparison Figure 6. Predicted results for the rational (middit
with human data (top row) for three experiments.
Human Data

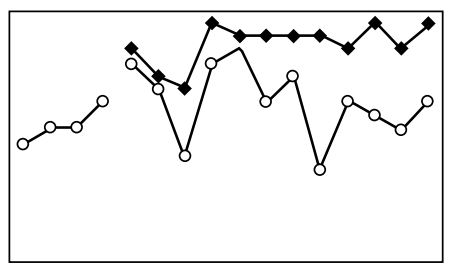

Rational Model

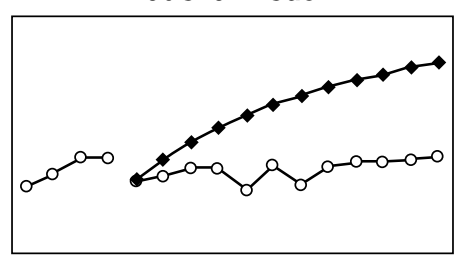

Autoassociation Model
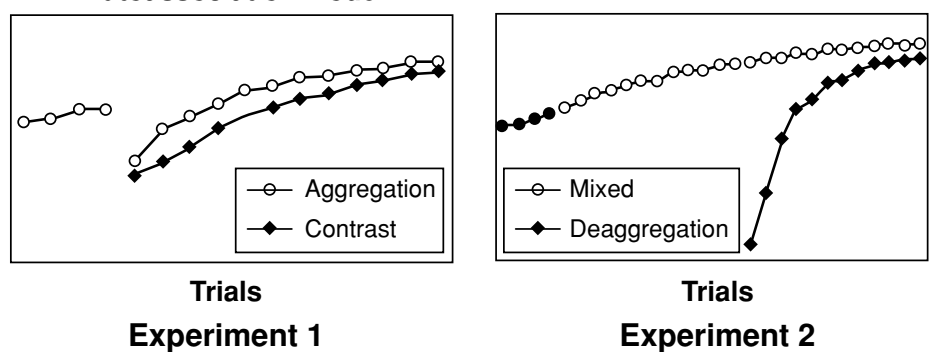
model never has the opportunity to learn the predictive structure within these categories. Thus, the rational model predicts that Category B will be learned better in the contrast condition despite the eight extra B instances shown in the mixed condition.

McClelland and Rumelhart's (1985) autoassociation model, represented in the bottom panel of the column, produces a very different pattern of results given the same training sequences. This model does predict some interference when two categories are mixed together, relative to a condition in which a single category is presented alone. For example, Category A is learned faster during the first 16 trials of the contrast condition, when it is shown alone, than in the mixed condition, when it is shown together with Category B (note that Category A data are not shown in Figure 6). However, no violation of monotonicity is predicted: Learning of Category B in the contrast condition remains lower than that in the mixed condition throughout the experiment. Given enough trials, both reach approximately the same asymptote.

In the middle column, predictions of the two models are compared with the human data from Experiment 1 of the present article. In this experiment, adding examples from both categories to the beginning of a standard contrast sequence reduced overall learning. Once again, the rational model is able to reproduce the main qualitative features of the human data whereas the autoassociation model is not. In the aggregation condition, the rational model begins by aggregating the two categories and then fails to deaggregate them during the later series of A instances, resulting in a reduction in predicted recall relative to the contrast condition. The autoassociation model predicts the opposite result - that is, that adding more examples of a pattern at the beginning of training should strengthen that pattern (contrary to what is observed in the human data).

The right column shows the results for the present Experiment 2. The rational model (middle panel) captures the qualitative pattern of the human data (top panel) quite well, although the model does require a slightly longer series of $\mathrm{A}$ instances to deaggregate the categories. ${ }^{2}$ The autoassociation model (bottom panel) once again fails to match the human data. According to this model, the B pattern continues to be strengthened during the target interval in the mixed condition but not in the deaggregation condition (in which no Bs are shown during this interval). Due to the larger number of examples shown in the mixed condition, final learning of $\mathrm{B}$ is expected to be greater in that condition than in the deaggregation condition (contrary to the actual data).

\section{Conclusions and Implications}

In sum, the rational model provides a very good qualitative fit to the data whereas the autoassociation model fits the data very poorly. Obviously, this outcome has implications for these particular models and possibly for the larger classes of models to which they belong. A key question in evaluating these larger implications is the extent to which the behavior of these two specific models can be taken as representative of correlation tracking versus category in- vention models in general. The answer to this question depends on determining which characteristics of the models are responsible for their ability (or inability) to reproduce the patterns of learning observed in the human data.

Regarding the rational model, the main characteristics that enable it to accommodate the present results (aside from its ability to invent categories) are its variable parameters, which allow the model to simulate a learner with relatively poor memory who is not actively searching for new categories (Clapper \& Bower, 2002). This enables the model to reproduce seemingly "irrational" or "suboptimal" aspects of human learning by taking human limitations into account in a realistic manner (i.e., the current patterns of learning may actually be optimal, given appropriate assumptions about people's memory limitations and learning biases). Category invention models that do not share these features (e.g., those that are designed always to capture as much of the predictive structure of a domain as possible, to be relatively insensitive to the order in which training instances are presented, and to lack adjustable parameters that allow them to accommodate human limitations) would be unable to account for the present results.

Unlike the rational model, the McClelland and Rumelhart (1985) model was unable to capture the main qualitative features of the human data. This model has a single learning process (adjusting connection strengths), and that process is necessarily incremental and varies as a monotonic function of exposure. In fact, all the models referred to here as correlation tracking models share this strategy of incremental adjustment, and hence all are committed to monotonic learning. Given this fact, the present results must be taken as evidence against correlation tracking models as a class, not just against the specific autoassociation model examined here.

Of course, these results should not be interpreted as evidence against connectionist models in general, only against those restricted to autoassociation among preexisting stimulus features. In fact, there are a number of models that implement category invention within connectionist networks, such as the competitive learning model of Rumelhart and Zipser (1986) and the SUSTAIN model of Love et al. (2004). Typically, these models consist of two or more layers or levels, with higher levels used to represent categories at lower levels. It should also be noted that autoassociation models themselves (see, e.g., Kohonen et al., 1981; McClelland \& Rumelhart, 1985) often assume that the overall processing system consists of many separate modules or layers (even when the computational versions of those models implement only a single module). By assuming that units or patterns in higher level modules can be recruited to represent categories at lower levels, such models can implement a form of category invention (see, e.g., Hawkins \& Blakeslee, 2004). Thus, the present negative conclusions about autoassociation should be seen as applying to individual layers or modules, but not necessarily to complex, multilayered systems consisting of many such modules.

Metaphorically, the task of finding the "correct" model of human learning can be conceptualized as a process of 
searching through a vast space of possible alternatives and gradually narrowing that space down to more and more manageable dimensions. The fact that many different models are, in principle, compatible with any data set makes this task all the more challenging. Hence, science often makes progress not by showing that a particular model is necessarily correct, but rather by revealing which models are necessarily incorrect and establishing some of the essential features that any correct model must possess. The present results are especially useful in this regard because they eliminate an entire class of models, some of which are quite powerful and intuitively compelling in other respects. In addition, the present results show that any adequate model of unsupervised learning must have certain qualities; in particular, it must possess the ability to create new categories on the basis of perceived contrast or mismatch, and it must take human cognitive limitations into account. The nonincremental, dichotomous view of learning that emerges from these results has many implications, not only for computational models but also for general ideas regarding the nature of categories and how they are learned in everyday life. It is hoped that the methods employed here will contribute to further progress in exploring these issues.

\section{REFERENCES}

Anderson, J. A. (1977). Neural models with cognitive implications. In D. LaBerge \& S. J. Samuels (Eds.), Basic processes in reading: Perception and comprehension (pp. 27-90). Hillsdale, NJ: Erlbaum.

Anderson, J. A., Silverstein, J. W., Ritz, S. A., \& Jones, R. S. (1977). Distinctive features, categorical perception, and probability learning: Some applications of a neural model. Psychological Review, 84, 413-451.

ANDERSON, J. R. (1990). The adaptive character of thought. Hillsdale, NJ: Erlbaum.

ANDERSON, J. R. (1991). The adaptive nature of human categorization. Psychological Review, 98, 409-429.

BaIn, A. (1968). The senses and the intellect. New York: Appleton. (Original work published 1855)

Billman, D., \& Heit, E. (1988). Observational learning from internal feedback: A simulation of an adaptive learning method. Cognitive Science, 12, 587-625.

Billman, D., \& Knutson, J. (1996). Unsupervised concept learning and value systematicity: A complex whole aides learning the parts. Journal of Experimental Psychology: Learning, Memory, \& Cognition, 22, 458-475.

Bruner, J. S., Goodnow, J. J., \& Austin, G. A. (1967). A study of thinking. New York: Wiley.

Clapper, J. P., \& Bower, G. H. (1991). Learning and applying category knowledge in unsupervised domains. In G. H. Bower (Ed.), The psychology of learning and motivation (Vol. 27, pp. 65-108). New York: Academic Press.

Clapper, J. P., \& Bower, G. H. (1994). Category invention in unsupervised learning. Journal of Experimental Psychology: Learning, Memory, \& Cognition, 20, 443-460.

Clapper, J. P., \& Bower, G. H. (2002). Adaptive categorization in unsupervised learning. Journal of Experimental Psychology: Learning, Memory, \& Cognition, 28, 908-923.

DAVIs, B. R. (1985). An associative hierarchical self-organizing system. IEEE Transactions on Systems, Man, \& Cybernetics, SMC-15, 570579.

Gureckis, T. M., \& Love, B. C. (2003). Towards a unified account of supervised and unsupervised category learning. Journal of Experimental \& Theoretical Artificial Intelligence, 15, 1-24.

Hawkins, J., \& BlakesleE, S. (2004). On intelligence. New York: Holt.
Hume, D. A. (2000). A treatise of human nature. New York: Oxford University Press. (Original work published 1739)

Kohonen, T., OJA, E., \& Lehtio, P. (1981). Storage and processing of information in distributed associative memory systems. In G. E. Hinton \& J. A. Anderson (Eds.), Parallel models of associative memory (pp. 104-143). Hillsdale, NJ: Erlbaum.

Love, B. C., Medin, D. L., \& Gureckis, T. M. (2004). SUSTAIN: A network model of category learning. Psychological Review, 111, 309332

Malt, B. C., Ross, B. H., \& Murphy, G. L. (1995). Predicting features for members of natural categories when categorization is uncertain. Journal of Experimental Psychology: Learning, Memory, \& Cognition, 21, 646-661.

McClelland, J. L., \& Rumelhart, D. E. (1985). Distributed memory and the representation of general and specific information. Journal of Experimental Psychology: General, 114, 159-188.

Medin, D. L., \& Schaffer, M. M. (1978). Context theory of classification learning. Psychological Review, 85, 207-238.

Mervis, C. B. (1987). Child-basic object categories and early lexical development. In U. Neisser (Ed.), Concepts and conceptual development: Ecological and intellectual factors in categorization (pp. 201233). Cambridge: Cambridge University Press.

Metcalfe, J. (2002). Is study time allocated selectively to a region of proximal learning? Journal of Experimental Psychology: General, 131, 349-363.

Murphy, G. L. (2002). The big book of concepts. Cambridge, MA: MIT Press.

Murphy, G. L., \& Ross, B. H. (1994). Predictions from uncertain categorizations. Cognitive Psychology, 27, 148-193.

Nelson, K. (1974). Concept, word, and sentence: Interrelations in acquisition and development. Psychological Review, 81, 267-285.

Posner, M. I., \& KeELe, S. W. (1968). On the genesis of abstract ideas. Journal of Experimental Psychology, 77, 353-363.

Posner, M. I., \& KeEle, S. W. (1970). Retention of abstract ideas. Journal of Experimental Psychology, 83, 304-308.

Rumelhart, D. E., \& ZiPSER, D. (1986). Feature discovery by competitive learning. In J. L. McClelland \& D. E. Rumelhart (Eds.), Parallel distributed processing: Explorations in the microstructure of cognition (Vol. 1, pp. 151-193). Cambridge, MA: MIT Press.

Schank, R. C. (1982). Dynamic memory: A theory of reminding and learning in computers and people. Cambridge: Cambridge University Press.

Smith, E. E., \& Medin, D. L. (1981). Categories and concepts. Cambridge, MA: Harvard University Press.

Sutton, R. S., \& BARTo, A. G. (1981). Toward a modern theory of adaptive networks: Expectation and prediction. Psychological Review, 88, 135-170.

Trabasso, T., \& Bower, G. H. (1968). Attention in learning: Theory and research. New York: Wiley.

Zeaman, D., \& House, B. J. (1963). The role of attention in retardate discrimination learning. In N. R. Ellis (Ed.), Handbook of mental deficiency (pp. 159-223). New York: McGraw-Hill.

\section{NOTES}

1. In the original version of the rational model (J. R. Anderson, 1990, 1991), feature likelihoods were computed using a weighted sum of the value's conditional probability within all categories. However, results from Murphy and Ross (1994) and Malt, Ross, and Murphy (1995) suggested that only the most probable category is normally used in estimating feature probabilities. In the present case, the difference in outputs is negligible because the probabilities of nonselected categories, $P(k \mid F)$, are so low that taking them into account has no significant effect.

2. The training sequences used as inputs to the two computer models contained a series of $33 \mathrm{~A}$ instances, in contrast to the 24 used in Experiment 2 . This is the approximate minimum required by the rational model to deaggregate the categories following an initial mixed sequence, given the parameter values assumed here.

(Manuscript received September 9, 2004; revision accepted for publication May 31, 2005.) 